\title{
DC Programming for Power Minimization in a Multicell Network with RF-Powered Relays
}

\author{
Ali A. Nasir, Duy T. Ngo, and Salman Durrani \\ Emails: ali.nasir@anu.edu.au,duy.ngo@newcastle.edu.au, and salman.durrani@anu.edu.au,
}

\begin{abstract}
We consider a multicell network where an amplifyand-forward relay is deployed in each cell to help the base station (BS) serve its cell-edge user. We assume that each relay scavenges energy from all received radio signals to process and forward the information data from the BS to the corresponding user. For this, a power splitter and a wireless energy harvester are implemented in the relay. Our aim is to minimize the total power consumption in the network while guaranteeing minimum data throughput for each user. To this end, we develop a resource management scheme that jointly optimizes three parameters, namely, BS transmit powers, power splitting factors for energy harvesting and information processing at the relays, and relay transmit powers. As the formulated problem is highly nonconvex, we devise a successive convex approximation algorithm based on differenceof-convex-functions (DC) programming. The proposed iterative algorithm transforms the nonconvex problem into a sequence of convex problems, each of which is solved efficiently in each iteration. We prove that this path-following algorithm converges to an optimal solution that satisfies the Karush-Kuhn-Tucker (KKT) conditions of the original nonconvex problem. Simulation results demonstrate that the proposed joint optimization solution substantially improves the network performance.
\end{abstract}

\section{INTRODUCTION}

In a multicell network, users at the cell edges are the victims of strong intercell interference while receiving weak signals from their serving base stations (BSs). A viable solution is to deploy relay nodes to provide network coverage to the celledge users [1]. While connecting to the BSs via wireless links, the opportunistic nature of relay deployments may restrict their access to a main power supply. This problem can be solved by implementing wireless energy harvesting techniques at the relays, where energy is scavenged from the ambient propagating electromagnetic waves in the radio frequency (RF) [2]-[4]. Wireless energy harvesting solutions are feasible for relays, which only require significantly low transmit power due to their restricted network coverage.

With RF-powered relays, the network performance is determined by the transmit powers allocated to the BSs and the relays, as well as the use of RF energy harvested at the relays. The work of [5] proposes coordinated scheduling and power control algorithms only for the BSs of a multicell network. The study of [6] develops resource allocation schemes specifically for the remote radio heads. Reference [7] studies the power control problem for multiuser broadband wireless systems without relays, where simultaneous wireless information and power transfer is assumed. An optimal power splitting rule is

Ali A. Nasir and Salman Durrani are with the Research School of Engineering, CECS, The Australian National University, Canberra, Australia, and Duy. T. Ngo is with School of Electrical Engineering and Computer Science, University of Newcastle, Australia. The work of A. A. Nasir and S. Durrani was supported in part by the Australian Research Council's Discovery Project funding scheme (project number DP140101133). The work of D. T. Ngo was supported in part by the University of Newcastle's Early Career Researcher Grant G1301282. devised in [8] for energy harvesting and information processing at the RF-powered relays of a multiuser interference network.

This paper considers a multicell network where an energyconstrained relay node assists the BS of each cell to communicate with its cell-edge user. Equipped with a wireless energy harvester, the relay is capable of scavenging part of the RF energy in the received signal. A power splitter is included in the relay to help decide how much received signal energy is to be harvested. The information transceiver at the relay will use the harvested energy to amplify and then forward the BS signal to its corresponding user.

We aim to devise an optimal scheme to manage the radio resources at the BSs and RF-powered relays. The objective is to minimize the total transmit power of all BSs, subject to meeting a minimum data rate for each user. This problem is of particular interest for "green" communications, where one wishes to reduce the environmental impacts of largescale wireless network deployment while providing quality of service to the most vulnerable users. Different from existing works, we jointly optimize the transmit powers at the BSs and the relays, together with finding an optimal power splitting rule for energy harvesting and signal processing at the relays. The strongly-coupled optimizing variables render the formulated problem highly nonconvex, and thus challenging to solve.

In this work, we employ difference-of-convex-functions (DC) programming and apply the successive convex approximation (SCA) method to transform the nonconvex problem to a series of convex programs [9], [10]. Specifically, we express the nonconvex throughput function as the difference of two convex functions and apply the first-order Taylor series approximation to convexify it. We then propose an iterative algorithm where only one simple convex problem is to be solved at each iteration. We prove that the DC-based SCA algorithm generates a sequence of feasible and improved solutions, which monotonically converges to solutions satisfying the KKT conditions of the original nonconvex problem. While a true globally optimal method does not exist for the formulated problem, it has been shown that the SCA-based solutions often empirically achieve the global optimum in most practical scenarios [11], [12].

\section{System Model AND Problem Formulation}

We consider the downlink of a multicell network with universal frequency reuse, as illustrated in Fig. 1. Let $\mathcal{N}=$ $\{1, \ldots, N\}$ denote the set of all cells. We assume that the BSs are connected to a central processing (CP) unit which coordinates the multicell transmissions and radio resource management. While the BS of cell $i \in \mathcal{N}$ attempts to communicate with its single user, this user is located at the cell edge and hence out of reach. A relay node is deployed in each cell to provide network coverage, assisting the communication 


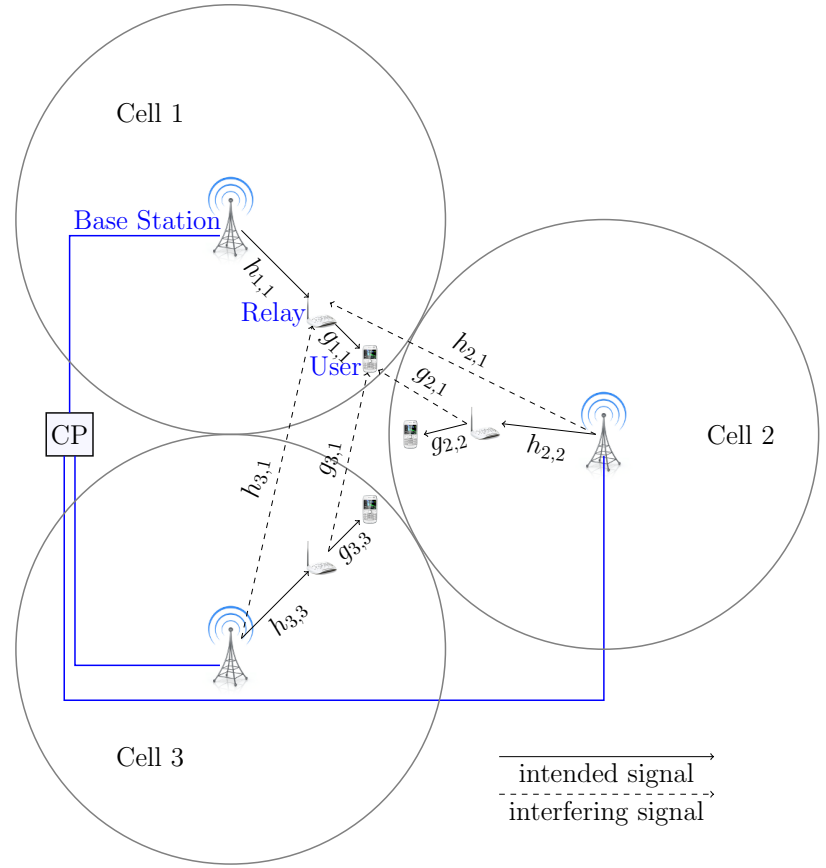

Fig. 1. Illustration of a multicell network with 3 cells. While only the interfering scenario is shown for cell 1 , the interference actually occurs at the receivers of all three relays and three users.

from the BS to its user. Denote the channel coefficient from BS $i$ to relay $j$ as $h_{i, j}$, and that from relay $j$ to user $k$ as $g_{j, k}$. We assume that all BSs send the available channel state information to the CP unit via a dedicated control channel.

We assume that each of relays is equipped with a wireless energy harvester that scavenges energy in the received RF signals from all BSs. The harvested energy is used by an information transceiver at the relay to forward the message signal to the intended user. We divide the total transmission block time $T$ into two equal time slots as illustrated in Fig. 2.

\section{A. BS-to-Relay Transmissions and Wireless Energy Harvesting at Relays}

The first time slot $[0, T / 2]$ includes BS-to-relay transmissions and RF energy harvesting at the relays. This is done while all the relays do not transmit. Let $x_{i}$ denote the normalized information signal sent by BS $i$, i.e., $\mathbb{E}\left\{\left|x_{i}\right|^{2}\right\}=1$. Let $P_{i}$ be the transmit power of BS $i, d_{i, j}^{h}$ the distance between BS $i$ and relay $j$, and $\beta$ the path-loss exponent. Assume that $n_{i}^{a}$ is the zero-mean additive white Gaussian noise (AWGN) with variance $\sigma_{i}^{a}$ at the receive antenna of relay $i$. The received signal at relay $i$ is expressed as

$$
y_{R_{i}}=\bar{h}_{j, i} \sqrt{P_{i}} x_{i}+\sum_{j=1, j \neq i}^{N} \bar{h}_{j, i} \sqrt{P_{j}} x_{j}+n_{i}^{a},
$$

where $\bar{h}_{j, i} \triangleq h_{j, i}\left(d_{j, i}^{h}\right)^{-\beta / 2}$ is the effective channel gain from BS $j$ to relay $i$.

To implement dual energy harvesting and signal processing at the relays, each relay is equipped with a power splitter that specifies how much received signal energy is dedicated to each of the two purposes [2], [8]. Fig. 2 shows that the power splitter at relay $i \in \mathcal{N}$ divides the power of $y_{R_{i}}$ into two parts in the proportion of $\alpha_{i}:\left(1-\alpha_{i}\right)$, where $\alpha_{i} \in(0,1)$ is the power splitting factor. The first part $\sqrt{\alpha_{i}} y_{R_{i}}$ is processed by the energy harvester and stored as energy (e.g., by charging a battery at relay $i$ ) for use in the second time slot. The amount of energy harvested at relay $i$ is given by

$$
E_{i}=\frac{\eta \alpha_{i} T}{2} \sum_{j=1}^{N} P_{j}\left|\bar{h}_{j, i}\right|^{2},
$$

where $\eta \in(0,1)$ denotes the efficiency of energy conversion.

The second part $\sqrt{1-\alpha_{i}} y_{R_{i}}$ of the received signal is passed to an information transceiver. In Fig. $2, n_{i}^{r}$ denotes the AWGN with zero mean and variance $\sigma_{i}^{r}$ introduced by the baseband processing circuitry. Since antenna noise power $\sigma_{i}^{a}$ is very small compared to the circuit noise power $\sigma_{i}^{r}$ in practice [13], $n_{i}^{a}$ has a negligible impact on both the energy harvester and the information transceiver of relay $i$. For simplicity, we will thus ignore the effect of $n_{i}^{a}$ by setting $\sigma_{i}^{a}=0$. The signal at the input of the information transceiver of relay $i$ is then expressed as

$$
\begin{aligned}
y_{R_{i}}^{I} & =\sqrt{1-\alpha_{i}} y_{R_{i}}+n_{i}^{r} \\
& =\sqrt{1-\alpha_{i}} \bar{h}_{i, i} \sqrt{P_{i}} x_{i}+\sqrt{1-\alpha_{i}} \sum_{j=1, j \neq i}^{N} \bar{h}_{j, i} \sqrt{P_{j}} x_{j}+n_{i}^{r}
\end{aligned}
$$

where the first term in (3) is the desired signal from BS $i$, and the second term is the total interference from all other BSs.

B. Signal Processing at Relays and Relay-to-User Transmissions

The second time slot $[T / 2, T]$ includes signal processing at the relays and relay-to-user transmissions. This is done while all the BSs suppress their transmissions. Here, the information transceiver amplifies the signal $y_{R_{i}}^{I}$ before forwarding it to user $i$. Let $p_{i}$ denote the transmit power of relay transceiver $i$. From (2), it is clear that

$$
p_{i} \leq \frac{E_{i}}{T / 2}=\eta \alpha_{i} \sum_{j=1}^{N} P_{j}\left|\bar{h}_{j, i}\right|^{2} .
$$

The transmitted signal from relay $i$ to user $i$ is given by

$$
x_{R_{i}}=\zeta_{i} \sqrt{p_{i}} y_{R_{i}}^{I},
$$

where $\zeta_{i} \triangleq\left[\left(1-\alpha_{i}\right) \sum_{j=1}^{N} P_{j} \bar{h}_{j, i}+\sigma_{i}^{r}\right]^{1 / 2}$ is the amplifying factor that ensures power constraint in (4) be met.

The received signal at user $i$ is then

$$
y_{U_{i}}=\bar{g}_{i, i} x_{R_{i}}+\sum_{j=1, j \neq i}^{N} \bar{g}_{j, i} x_{R_{j}}+n_{i}^{u},
$$

where $\bar{g}_{j, i} \triangleq g_{j, i}\left(d_{j, i}^{g}\right)^{-\beta / 2}$ is the effective channel gain from BS $j$ to relay $i, d_{i, j}^{g}$ the distance between relay $i$ and user $j$, and $n_{i}^{u}$ the AWGN with zero mean and variance $\sigma_{i}^{u}$ at the receiver of user $i$. Using $x_{R_{i}}$ in (5) and $y_{R_{i}}^{I}$ in (3), we can rewrite (6) as

$$
\begin{aligned}
y_{U_{i}}=\zeta_{i} \bar{g}_{i, i} \bar{h}_{i, i} \sqrt{p_{i} P_{i}\left(1-\alpha_{i}\right)} x_{i} \\
+\zeta_{i} \bar{g}_{i, i} \sqrt{p_{i}\left(1-\alpha_{i}\right)} \sum_{j=1, j \neq i}^{N} \bar{h}_{j, i} \sqrt{P_{j}} x_{j} \\
+\zeta_{i} \bar{g}_{i, i} \sqrt{p_{i}} n_{i}^{r}+\sum_{j=1, j \neq i}^{N} \zeta_{j} \bar{g}_{j, i} \sqrt{p_{j}} y_{R_{j}}^{I}+n_{i}^{u} .
\end{aligned}
$$




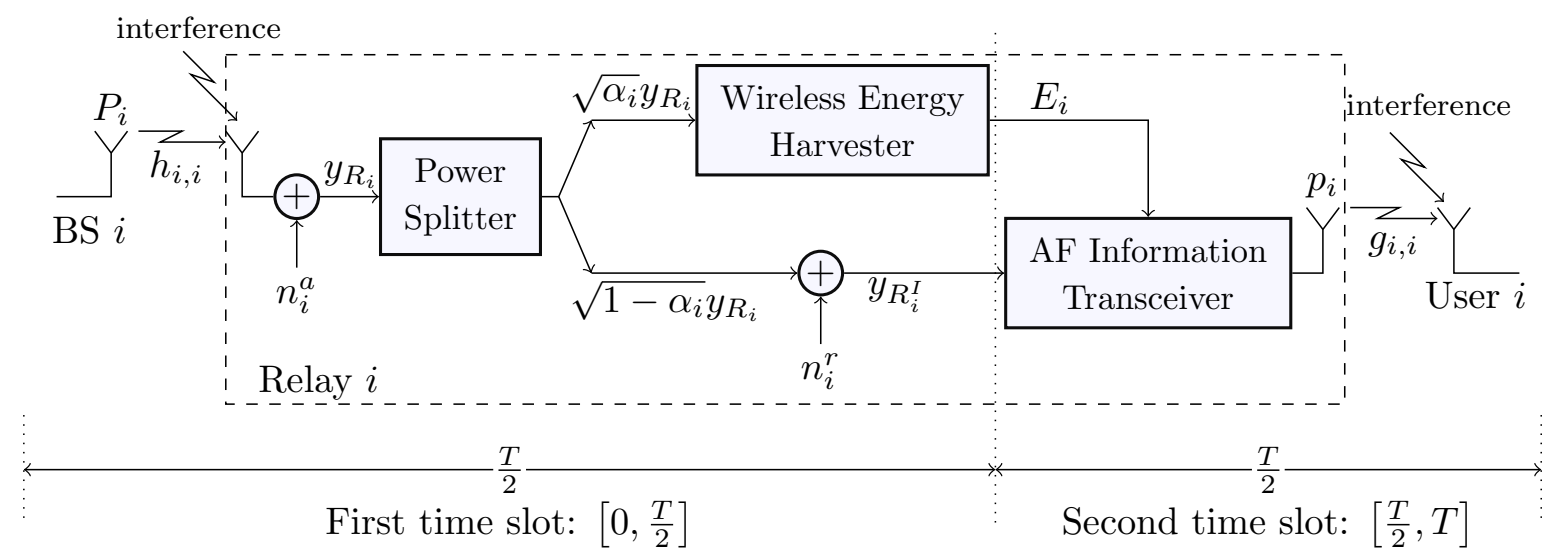

Fig. 2. BS-to-user communication assisted by an RF-powered relay.

The first term in (7) is the signal from BS $i$ to its user $i$, and the remaining terms are the total intercell interference and noise.

Without loss of generality, let us assume $\sigma_{i}^{r}=\sigma_{i}^{u}=\sigma, \forall i \in$ $\mathcal{N}$. From (7), the signal-to-interference-plus-noise ratio (SINR) at the receiver of user $i$ is given in (8) at the bottom of this page, where we define

$$
\begin{aligned}
& \phi_{1}^{i, j} \triangleq \frac{\left|\bar{g}_{i, i} \bar{h}_{j, i}\right|^{2}}{\sigma^{2}} ; \quad \phi_{2}^{i, j} \triangleq \frac{\left|\bar{h}_{j, i}\right|^{2}}{\sigma} ; \\
& \phi_{3}^{i, j} \triangleq \frac{\left|\bar{g}_{j, i}\right|^{2}}{\sigma} ; \quad \phi_{4}^{i, j, k} \triangleq \frac{\left|\bar{g}_{j, i} \bar{h}_{k, i}\right|^{2}}{\sigma^{2}} .
\end{aligned}
$$

For notational convenience, let us also define $\mathbf{P} \triangleq$ $\left[P_{1}, \ldots, P_{N}\right]^{T}, \mathbf{p} \triangleq\left[p_{1}, \ldots, p_{N}\right]^{T}$, and $\boldsymbol{\alpha} \triangleq\left[\alpha_{1}, \ldots, \alpha_{N}\right]^{T}$. From (8), the achieved throughput of cell $i$ is given as

$$
\tau_{i}(\mathbf{P}, \mathbf{p}, \boldsymbol{\alpha})=\frac{1}{2} \log _{2}\left(1+\gamma_{i}\right)
$$

It is observed from (8) and (10) that if we increase $\alpha_{i}$ (i.e., dedicate more received power for energy harvesting at relay $i$ ), the end-to-end throughput $\tau_{i}$ of cell $i$ might be degraded. This can be verified upon dividing both the numerator and the denominator of $\gamma_{i}$ in (8) by $\left(1-\alpha_{i}\right)$. If we instead decrease $\alpha_{i}$, the transmit power available at the information transceiver of relay $i$ in (4) will be further limited, thus potentially reducing $\tau_{i}$. Similarly, increasing $P_{i}$ or $p_{i}$ does not necessarily enhance $\tau_{i}$ because these two parameters appear in the positive terms at both the numerator and the denominator of $\gamma_{i}$.

In this paper, we aim to develop a resource allocation scheme that delivers an optimal tradeoff among transmit power $\mathbf{P}$ at the BSs, transmit power $\mathbf{p}$ at the relays, and power splitting factor $\boldsymbol{\alpha}$ at the relays. Our objective is to minimize the total transmit power from the BSs, while assuring a minimum data throughput $\tau_{\text {min }}$ for each user. Other resource allocation problems are considered in [14]. The design problem is formulated as follows:

$$
\begin{aligned}
\min _{\mathbf{P}, \mathbf{p}, \boldsymbol{\alpha}} & \sum_{i=1}^{N} P_{i} \\
\text { s.t. } & \tau_{i} \geq \tau_{\min }, \quad \forall i \in \mathcal{N} \\
& 0 \leq \alpha_{i} \leq 1, \quad \forall i \in \mathcal{N} \\
& P_{\min } \leq P_{i} \leq P_{\max }, \quad \forall i \in \mathcal{N} \\
& 0 \leq p_{i} \leq \eta \alpha_{i} \sum_{j=1}^{N} P_{j}\left|\bar{h}_{j, i}\right|^{2}, \quad \forall i \in \mathcal{N},
\end{aligned}
$$

where $P_{\max }$ denotes the maximum power available for transmission at each $\mathrm{BS}$ and $P_{\min }$ is the minimum transmit power required at each $\mathrm{BS}$ to ensure the activation of energy harvesting circuitry at the relay. Constraint (11b) is imposed to protect the throughput performance of all cell-edge users. Constraint (11c) is for the power splitting factors at all relays, whereas constraints (11d) and (11e) ensure that the transmit powers at the BSs and relays do not exceed the maximum limit.

Problem (11) is nonconvex in $(\mathbf{P}, \mathbf{p}, \boldsymbol{\alpha})$ because the throughput $\tau_{i}$ in (11b) is nonconvex in these three variables. Even if we fix $\mathbf{p}$ and $\boldsymbol{\alpha}$ and try to optimize $\mathbf{P}$ alone, $\tau_{i}$ would still be highly nonconvex in $\mathbf{P}$ due to the cross-cell interference terms. Simultaneously optimizing $\mathbf{P}, \mathbf{p}$ and $\boldsymbol{\alpha}$ is much more challenging due to the nonlinearity introduced by the crossmultiplying terms, e.g., $P_{k} p_{j} \alpha_{i}$ in (8) and $\alpha_{i} P_{j}$ in (11e).

\section{Proposed SCA Solution Using DC PROGRAMMing}

We propose to adopt the successive convex approximation (SCA) approach [11], [12], [14] combined with DC programming [9], [10] to transform the nonconvex problem (11) into a sequence of convex subproblems. First, we will convexify the nonconvex constraints (11b) and (11e). Let us start with (11b) and rewrite the throughput expression $\tau_{i}$ in (10) as:

$$
\tau_{i}=\frac{1}{2}\left(v_{i}(\mathbf{x})-u_{i}(\mathbf{x})\right)
$$

$$
\gamma_{i}=\frac{\phi_{1}^{i, i} P_{i} p_{i}\left(1-\alpha_{i}\right)}{\sum_{j=1, j \neq i}^{N} \phi_{1}^{i, j} P_{j} p_{i}\left(1-\alpha_{i}\right)+\sum_{j=1}^{N}\left(\phi_{2}^{i, j} P_{j}\left(1-\alpha_{i}\right)+\phi_{3}^{i, j} p_{j}\right)+\sum_{j=1, j \neq i}^{N} \sum_{k=1}^{N} \phi_{4}^{i, j, k} P_{k} p_{j}\left(1-\alpha_{i}\right)+1}
$$




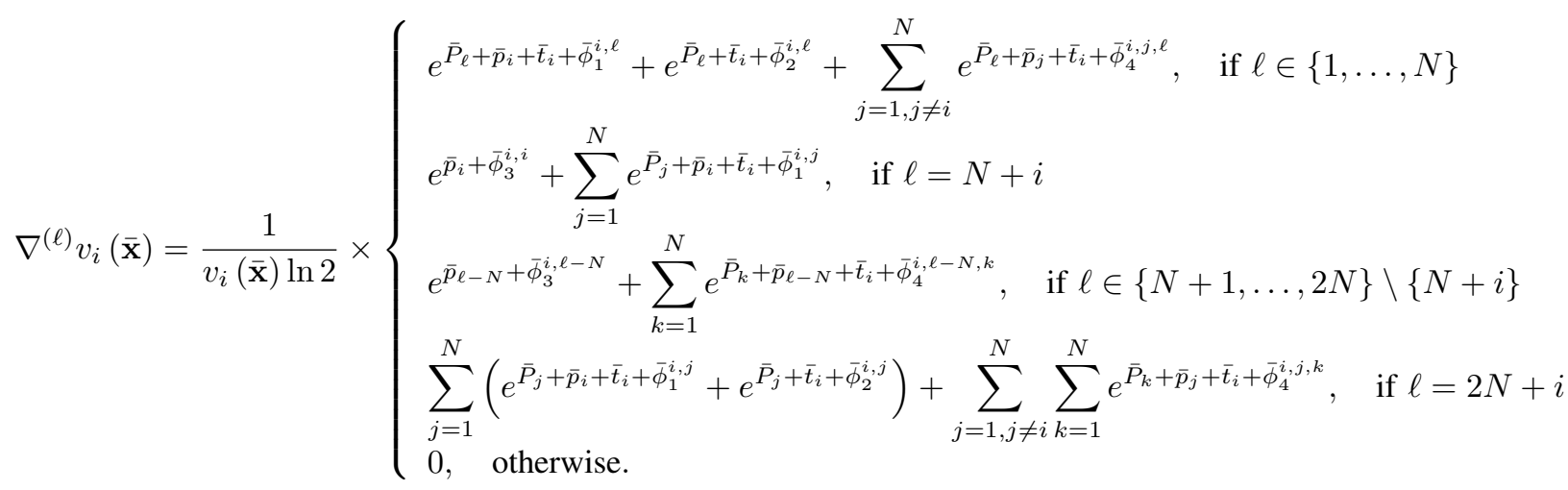

where $\mathbf{x}=\left[\mathbf{P}^{T}, \mathbf{p}^{T}, \mathbf{t}^{T}\right]^{T} \in \mathbb{R}_{+}^{3 N}, \mathbf{t}=\mathbf{1}-\boldsymbol{\alpha} \in \mathbb{R}_{+}^{N}$, and

$$
\begin{array}{r}
u_{i}(\mathbf{x}) \triangleq \log _{2}\left(\sum_{j=1, j \neq i}^{N} \phi_{1}^{i, j} P_{j} p_{i} t_{i}+\sum_{j=1}^{N}\left(\phi_{2}^{i, j} P_{j} t_{i}+\phi_{3}^{i, j} p_{j}\right)\right. \\
\left.+\sum_{j=1, j \neq i}^{N} \sum_{k=1}^{N} \phi_{4}^{i, j, k} P_{k} p_{j} t_{i}+1\right), \\
v_{i}(\mathbf{x}) \triangleq \log _{2}\left(\sum_{j=1}^{N}\left(\phi_{1}^{i, j} P_{j} p_{i} t_{i}+\phi_{2}^{i, j} P_{j} t_{i}+\phi_{3}^{i, j} p_{j}\right)\right. \\
\left.+\sum_{j=1, j \neq i}^{N} \sum_{k=1}^{N} \phi_{4}^{i, j, k} P_{k} p_{j} t_{i}+1\right) .
\end{array}
$$

Using the following logarithmic change of variables:

$$
\begin{aligned}
& \bar{P}_{i} \triangleq \ln P_{i} ; \quad \bar{p}_{i} \triangleq \ln p_{i} ; \quad \bar{t}_{i} \triangleq \ln t_{i} ; \quad \bar{\phi}_{1}^{i, j} \triangleq \ln \phi_{1}^{i, j} ; \\
& \bar{\phi}_{2}^{i, j} \triangleq \ln \phi_{2}^{i, j} ; \quad \bar{\phi}_{3}^{i, j} \triangleq \ln \phi_{3}^{i, j} ; \quad \bar{\phi}_{4}^{i, j, k} \triangleq \ln \phi_{4}^{i, j, k}
\end{aligned}
$$

for all $i, j, k \in \mathcal{N}$, we can further write $u_{i}(\cdot)$ and $v_{i}(\cdot)$ in terms of the sums of exponentials in $\overline{\mathbf{x}}$ :

$$
\begin{aligned}
u_{i}(\overline{\mathbf{x}})=\log _{2}\left(\sum_{j=1, j \neq i}^{N} e^{\bar{P}_{j}+\bar{p}_{i}+\bar{t}_{i}+\bar{\phi}_{1}^{i, j}}\right. & \\
& +\sum_{j=1}^{N}\left(e^{\bar{P}_{j}+\bar{t}_{i}+\bar{\phi}_{2}^{i, j}}+e^{\bar{p}_{j}+\bar{\phi}_{3}^{i, j}}\right) \\
& \left.+\sum_{j=1, j \neq i}^{N} \sum_{k=1}^{N} e^{\bar{P}_{k}+\bar{p}_{j}+\bar{t}_{i}+\bar{\phi}_{4}^{i, j, k}}+1\right),
\end{aligned}
$$

$v_{i}(\overline{\mathbf{x}})=\log _{2}\left(\sum_{j=1}^{N}\left(e^{\bar{P}_{j}+\bar{p}_{i}+\bar{t}_{i}+\bar{\phi}_{1}^{i, j}}+e^{\bar{P}_{j}+\bar{t}_{i}+\bar{\phi}_{2}^{i, j}}+e^{\bar{p}_{j}+\bar{\phi}_{3}^{i, j}}\right)\right.$

$$
\left.+\sum_{j=1, j \neq i}^{N} \sum_{k=1}^{N} e^{\bar{P}_{k}+\bar{p}_{j}+\bar{t}_{i}+\bar{\phi}_{4}^{i, j, k}}+1\right),
$$

where $\overline{\mathbf{x}} \triangleq\left[\overline{\mathbf{P}}^{T}, \overline{\mathbf{p}}^{T}, \overline{\mathbf{t}}^{T}\right]^{T}, \overline{\mathbf{P}} \triangleq\left[\bar{P}_{1}, \ldots, \bar{P}_{N}\right]^{T}, \overline{\mathbf{p}} \triangleq$ $\left[\bar{p}_{1}, \ldots, \bar{p}_{N}\right]^{T}$, and $\overline{\mathbf{t}} \triangleq\left[\bar{t}_{1}, \ldots, \bar{t}_{N}\right]^{T}$. Since the log-sum-exp function is convex [15], both $u_{i}(\overline{\mathbf{x}})$ and $v_{i}(\overline{\mathbf{x}})$ are convex in $\overline{\mathbf{x}}$. However, $v_{i}(\overline{\mathbf{x}})-u_{i}(\overline{\mathbf{x}})$ in $(12)$ is not necessarily concave.

Using the first-order Taylor series expansion around a given point $\overline{\mathbf{x}}^{[m-1]}$, we propose to approximate $v_{i}(\overline{\mathbf{x}})$ by an affine function as follows [10]:

$$
v_{i}(\overline{\mathbf{x}}) \approx v_{i}\left(\overline{\mathbf{x}}^{[m-1]}\right)+\left(\nabla v_{i}\left(\overline{\mathbf{x}}^{[m-1]}\right)\right)^{T}\left(\overline{\mathbf{x}}-\overline{\mathbf{x}}^{[m-1]}\right),
$$

where the $\ell$-th element of gradient $\nabla v_{i}(\overline{\mathbf{x}})$ is given by (19), at the top of this page. With the affine approximation (18) and the convex function $u_{i}(\overline{\mathbf{x}})$, it is clear that the constraint (11b) can now be approximated by a concave function as:

$v_{i}\left(\overline{\mathbf{x}}^{[m-1]}\right)+\left(\nabla v_{i}\left(\overline{\mathbf{x}}^{[m-1]}\right)\right)^{T}\left(\overline{\mathbf{x}}-\overline{\mathbf{x}}^{[m-1]}\right)-u_{i}(\overline{\mathbf{x}}) \geq 2 \tau_{\min }$.

Next, we convexify constraint (11e). By the variable change

$$
\bar{\alpha}_{i} \triangleq \ln \alpha_{i}, \quad \forall i \in \mathcal{N}
$$

and upon denoting $\overline{\boldsymbol{\alpha}} \triangleq\left[\bar{\alpha}_{1}, \ldots, \bar{\alpha}_{N}\right]^{T},(11 \mathrm{e})$ is rewritten as:

$$
e^{\bar{p}_{i}} \leq \eta e^{\bar{\alpha}_{i}} \sum_{j=1}^{N} e^{\bar{P}_{j}} \bar{h}_{j, i}
$$

Applying the arithmetic-geometric inequality, we have that:

$$
\sum_{j=1}^{N} e^{\bar{P}_{j}} \bar{h}_{j, i} \geq \prod_{j=1}^{N}\left(\frac{e^{\bar{P}_{j}} \bar{h}_{j, i}}{\lambda_{j, i}^{[m-1]}}\right)^{\lambda_{j, i}^{[m-1]}}
$$

where $\mathbf{P}^{[m-1]}$ is a fixed point and

$$
\lambda_{j, i}^{[m-1]} \triangleq \frac{e^{\bar{P}_{j}^{[m-1]}} \bar{h}_{j, i}}{\sum_{k=1}^{N} e^{\bar{P}_{j}^{[m-1]}} \bar{h}_{k, i}} .
$$

As such, (22) can be replaced by a stricter constraint:

$$
e^{\bar{p}_{i}} \leq \tilde{w}_{i}\left(\bar{\alpha}_{i}, \overline{\mathbf{P}}\right) \triangleq \eta e^{\bar{\alpha}_{i}} \prod_{j=1}^{N}\left(\frac{e^{\bar{P}_{j}} \bar{h}_{j, i}}{\lambda_{j, i}^{[m-1]}}\right)^{\lambda_{j, i}^{[m-1]}}
$$

which is equivalent to the following affine constraint:

$$
\bar{p}_{i}-\bar{\alpha}_{i}-\sum_{j=1}^{N} \lambda_{j, i}^{[m-1]} \bar{P}_{j}-c_{i} \leq 0
$$

where $c_{i} \triangleq \ln \eta+\sum_{j=1}^{N} \lambda_{j, i}^{[m-1]}\left(\ln \bar{h}_{j, i}-\ln \lambda_{j, i}^{[m-1]}\right)$ is a constant. From the convex approximations (20) and (26), we now have the following convex optimization problem which 


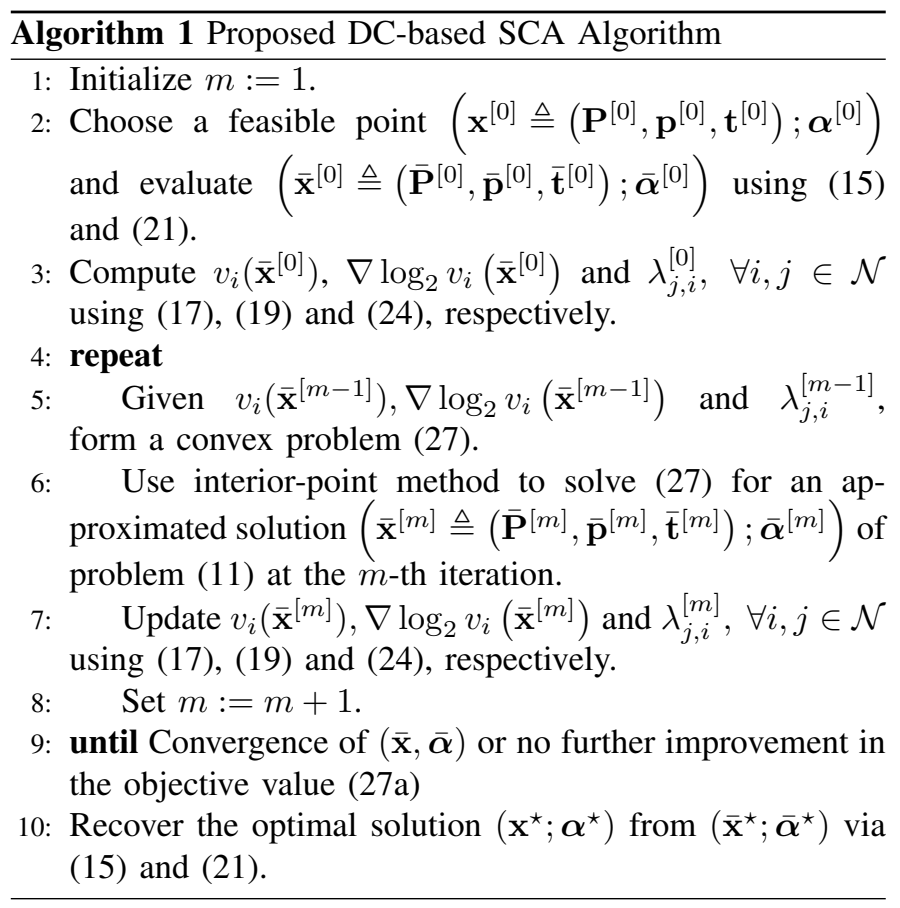

gives an approximated solution to problem (11) at the $m$-th iteration:

$$
\begin{aligned}
& \min _{\overline{\mathbf{x}}, \overline{\mathbf{\alpha}}} \sum_{i=1}^{N} P_{i} \\
& \text { s.t. } \quad v_{i}\left(\overline{\mathbf{x}}^{[m-1]}\right)+\left(\nabla v_{i}\left(\overline{\mathbf{x}}^{[m-1]}\right)\right)^{T}\left(\overline{\mathbf{x}}-\overline{\mathbf{x}}^{[m-1]}\right) \\
& -u_{i}(\overline{\mathbf{x}}) \geq 2 \tau_{\min }, \quad \forall i \in \mathcal{N} \\
& e^{\bar{t}_{i}}+e^{\bar{\alpha}_{i}} \leq 1, \quad \forall i \in \mathcal{N} \\
& e^{\bar{\alpha}_{i}} \leq 1, \quad \forall i \in \mathcal{N} \\
& e^{\bar{P}_{i}} \leq P_{\max }, \quad \forall i \in \mathcal{N} \\
& \bar{p}_{i}-\bar{\alpha}_{i}-\sum_{j=1}^{N} \lambda_{j, i}^{[m-1]} \bar{P}_{j}-c_{i} \leq 0, \quad \forall i \in \mathcal{N}
\end{aligned}
$$

where $\overline{\mathbf{x}}^{[m-1]}$ is known from the $(m-1)$-th iteration. In Algorithm 1, we propose an SCA algorithm in which a convex problem (27) is optimally solved at each iteration.

Proposition 1: Algorithm 1 generates a sequence of improved feasible solutions that converges to a point satisfying the KKT conditions of the original problem (11).

Proof: Since the gradient of the convex function $v_{i}(\overline{\mathbf{x}})$ is its subgradient [15], it follows that:

$$
v_{i}(\overline{\mathbf{x}}) \geq v_{i}\left(\overline{\mathbf{x}}^{[m-1]}\right)+\left(\nabla v_{i}\left(\overline{\mathbf{x}}^{[m-1]}\right)\right)^{T}\left(\overline{\mathbf{x}}-\overline{\mathbf{x}}^{[m-1]}\right) .
$$

This means that the approximated concave constraint $(27 \mathrm{~b})$ is stricter than the original nonconvex constraint (11b). Moreover, from (23) we have that $e^{\bar{p}_{i}} /\left(\eta e^{\bar{\alpha}_{i}} \sum_{j=1}^{N} e^{\bar{P}_{j}} \bar{h}_{j, i}\right) \leq$ $e^{\bar{p}_{i}} / \tilde{w}_{i}\left(\bar{\alpha}_{i}, \overline{\mathbf{P}}\right)$. This means that the approximated affine constraint $(27 \mathrm{f})$ is stricter than the original nonconvex constraint (11e). Due to the stricter constraints, the optimal solution of problem (27) will belong to the feasible set of problem (11).

If we initialize Algorithm 1 with a feasible point $\left(\overline{\mathbf{x}}^{[0]} ; \overline{\boldsymbol{\alpha}}^{[0]}\right)$, the presence of stricter constraints guarantees that the optimal solution $\left(\overline{\mathbf{x}}^{[m-1]} ; \overline{\boldsymbol{\alpha}}^{[m-1]}\right)$ to problem $(27)$ at the $(m-1)$ th iteration is a feasible solution to the same problem (27)

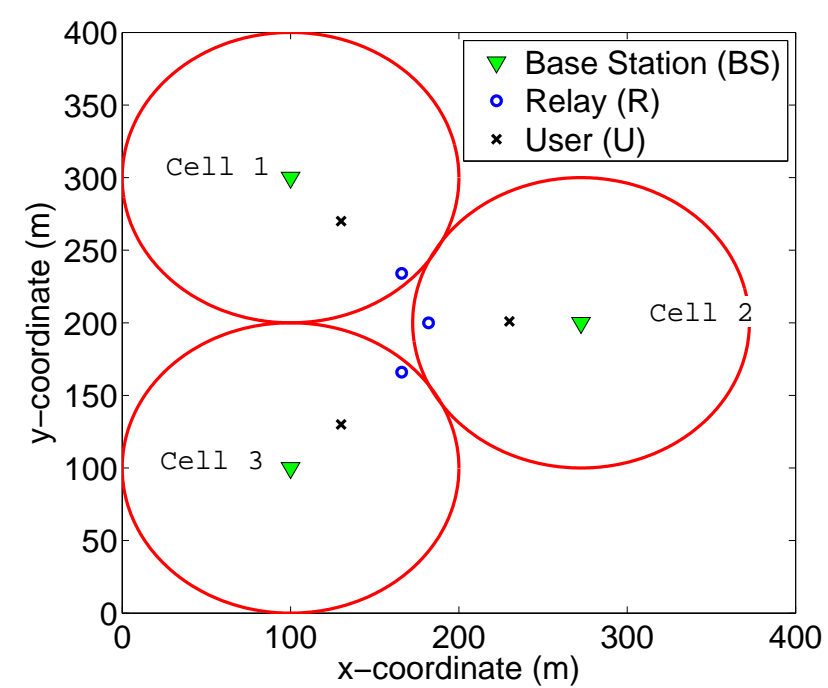

Fig. 3. The example multicell network used in the simulation.

at the $m$-th iteration. As such, the optimal value of problem (27) at the $m$-th iteration either improves or, at least, remains unchanged (by taking the optimal solution $\left(\overline{\mathbf{x}}^{[m-1]} ; \overline{\boldsymbol{\alpha}}^{[m-1]}\right)$ from the previous iteration). Algorithm 1 therefore converges to a point $\left(\overline{\mathbf{x}}^{\star} ; \overline{\boldsymbol{\alpha}}^{\star}\right)$.

Finally, it can be verified that

$$
\begin{aligned}
& \left.\nabla\left(v_{i}(\overline{\mathbf{x}})-u_{i}(\overline{\mathbf{x}})\right)\right|_{\overline{\mathbf{x}}=\overline{\mathbf{x}}^{[m-1]}}=\nabla\left(v_{i}\left(\overline{\mathbf{x}}^{[m-1]}\right)\right. \\
& \left.+\left(\nabla v_{i}\left(\overline{\mathbf{x}}^{[m-1]}\right)\right)^{T}\left(\overline{\mathbf{x}}-\overline{\mathbf{x}}^{[m-1]}\right)-u_{i}(\overline{\mathbf{x}})\right)\left.\right|_{\overline{\mathbf{x}}=\overline{\mathbf{x}}^{[m-1]}} \\
& \left.\nabla\left(\frac{e^{\bar{p}_{i}}}{\eta e^{\bar{\alpha}_{i}} \sum_{j=1}^{N} e^{\bar{P}_{j} \bar{h}_{j, i}}}\right)\right|_{\substack{\bar{\alpha}_{i}=\bar{\alpha}_{i}^{[m-1]} \\
\overline{\mathbf{P}}=\overline{\mathbf{P}}^{[m-1]}}}=\left.\nabla\left(\frac{e^{\bar{p}_{i}}}{\tilde{w}_{i}\left(\bar{\alpha}_{i}, \overline{\mathbf{P}}\right)}\right)\right|_{\substack{\bar{\alpha}_{i}=\bar{\alpha}_{i}^{[m-1]} \\
\overline{\mathbf{P}}=\overline{\mathbf{P}}^{[m-1]}}} .
\end{aligned}
$$

The results in (29)-(30) imply that the KKT conditions of the original problem (11) will be satisfied after the series of approximations involving convex problem (27) converges to $\left(\overline{\mathbf{x}}^{\star} ; \overline{\boldsymbol{\alpha}}^{\star}\right)$. This completes the proof.

\section{ILlustrative RESUlts}

Fig. 3 shows an example heterogeneous network where all cells have an equal cell radius of $100 \mathrm{~m}$. In cells 1 and 3 , we set the BS-relay and relay-user distances as $42 \mathrm{~m}$ and $51 \mathrm{~m}$, respectively. In cell 2 , the corresponding distances are $42 \mathrm{~m}$ and $48 \mathrm{~m}$. Note that the BS, relay and user in each cell do not lie on a straight line. We set the path loss exponent as $\beta=3$ and assume that the channel coefficients $h_{i, j}$ and $g_{j, k}, \forall i, j, k$ are circularly symmetric complex Gaussian random variables with zero mean and unit variance. With a block fading model, the randomly-generated values of $h_{i, j}$ and $g_{j, k}$ remain unchanged for the duration of resource allocation.

We fix BS transmit power budget at $P_{\max }=46 \mathrm{dBm}$. Recent research has shown that energy harvesting efficiency is practical in the range $0.4-0.6$ and minimum input power required to activate energy harvesting circuit is practical in the range $-10 \mathrm{dBm}$ to $-30 \mathrm{dBm}$ [16]. Hence we set $\eta=0.5$ and $P_{\text {min }}=26 \mathrm{dBm}$. These values ensure that for the assumed cell radius, the energy harvesting circuit is properly activated. With the channel bandwidth of $20 \mathrm{kHz}$ and the noise power density of $-174 \mathrm{dBm} / \mathrm{Hz}$, the total noise power is $\sigma=-131 \mathrm{dBm}$. We 


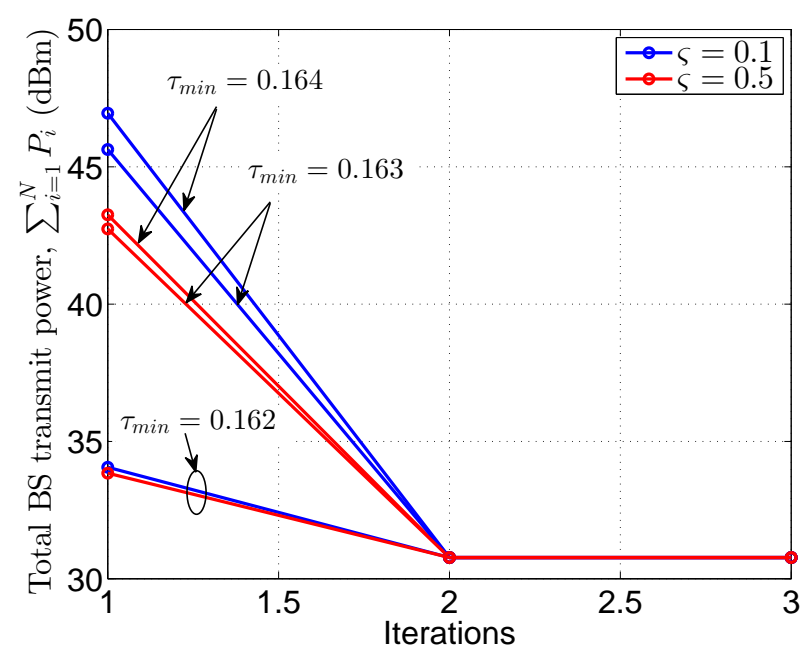

Fig. 4. Convergence process of Algorithm 1.

initialize Algorithm 1 with $P_{i}^{[0]}=\varsigma P_{\max } ; \alpha_{i}^{[0]}=\varsigma ; t_{i}^{[0]}=$ $1-\alpha_{i}^{[0]} ; \quad p_{i}^{[0]}=\varsigma \eta \alpha_{i}^{[0]} \sum_{j=1}^{N} P_{j}^{[0]} \bar{h}_{j, i}, \forall i \in \mathcal{N}$, where $\varsigma \in(0,1)$. To solve each convex problem in Algorithm 1, we resort to CVX, a package for specifying and solving convex programs [17].

Fig. 4 plots the convergence of the total BS power by Algorithm 1. Here, each iteration corresponds to solving of a problem (27) by CVX. It is clear from the figure that the proposed algorithm quickly converges in 3 iterations and the total required transmit power drops to the possible minimum, i.e., $N \times P_{\min }=30.7 \mathrm{dBm}$ for different values of $\tau_{\min }$.

It is not practical to compare the performance of Algorithm 1 with that of a globally optimal solution. There is no global optimization approach available in the literature to solve the highly nonconvex problem (11). A direct exhaustive search would incur a prohibitive computational complexity. However, Fig. 4 shows that initializing the algorithm with different values of $\varsigma$ does not affect the final solution. This means that the achieved throughput is insensitive to the initial points, further suggesting that the attained solution corresponds to the global optimum in our specific example [10], [11].

Fig. 5 demonstrates the advantage of jointly optimizing $(\mathbf{P}, \mathbf{p}, \boldsymbol{\alpha})$ as in the proposed Algorithm 1 over individual optimization of BS transmit power $\mathbf{P}$. In the latter approach, we fix $p_{i}=\eta \alpha_{i} \sum_{j=1}^{N} P_{j}$ and $\alpha_{i}=0.5, \forall i \in \mathcal{N}$. To obtain the results presented in the figure, we set $\varsigma=0.5$ and use the target minimum data rate $\tau_{\min }=\{0.161, \ldots, 0.165\}$. As seen, the proposed Algorithm always outperforms the sole optimization approach and 13-14 dB improvement is observed.

\section{CONCLUSIONS}

This paper has considered the problem of minimizing the total BS transmit power in a multicell network with RFpowered relays. We have attempted to jointly optimize the BS transmit powers, the relay power splitting factors and the relay transmit powers. To resolve the highly nonconvex problem formulation, we have proposed a successive convex approximation algorithm based on DC programming. We have proven that the devised algorithm converges to a locally optimal solution that satisfies the KKT conditions of the original nonconvex problem. Numerical examples have demonstrated the advantage of our approach.

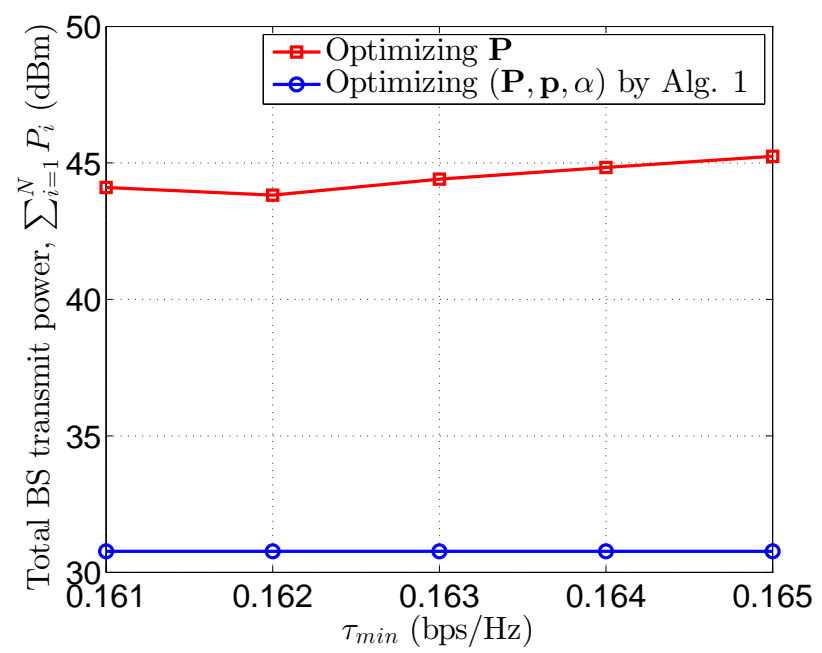

Fig. 5. Performance comparison of joint optimization Algorithm 1 and the sole optimization of $\mathbf{P}$.

\section{REFERENCES}

[1] Y. A. Sambo, M. Z. Shakir, K. A. Qaraqe, E. Serpedin, and M. A. Imran, "Expanding cellular coverage via cell-edge deployment in heterogeneous networks: Spectral efficiency and backhaul power consumption perspectives," IEEE Commun. Mag., vol. 52, no. 6, pp. 140-149, Jun. 2014.

[2] Z. Ding, S. M. Perlaza, I. Esnaola, and H. V. Poor, "Power allocation strategies in energy harvesting wireless cooperative networks," IEEE Trans. Wireless Commun., vol. 13, no. 2, pp. 846-860, Feb. 2014

[3] A. A. Nasir, X. Zhou, S. Durrani, and R. A. Kennedy, "Relaying protocols for wireless energy harvesting and information processing," IEEE Trans. Wireless Commun., vol. 12, no. 7, pp. 3622-3636, Jul. 2013.

[4] - "Wireless-powered relays in cooperative communications: Timeswitching relaying protocols and throughput analysis," ArXiv Technical Report, 2015. [Online]. Available: http://arxiv.org/abs/1310.7648

[5] N. Ksairi, P. Bianchi, and P. Ciblat, "Nearly optimal resource allocation for downlink OFDMA in 2-D cellular networks," IEEE Trans. Wireless Commun., vol. 10, no. 7, pp. 2101-2115, Jul. 2011.

[6] D. W. K. Ng and R. Schober, "Resource allocation for coordinated multipoint networks with wireless information and power transfer," in Proc. IEEE GLOBECOM, Austin, TX, USA, Dec. 2014.

[7] K. Huang and E. Larsson, "Simultaneous information and power transfer for broadband wireless systems," IEEE Trans. Signal Process., vol. 61, no. 23, pp. 5972-5986, Dec. 2013.

[8] H. Chen, Y. Li, Y. Jiang, Y. Ma, and B. Vucetic, "Distributed power splitting for SWIPT in relay interference channels using game theory," IEEE Trans. Wireless Commun., 2014, to appear. [Online]. Available: http://arxiv.org/abs/1408.3206

[9] P. Apkarian and H. D. Tuan, "Robust control via concave optimization: local and global algorithms," IEEE Trans. Autom. Control, vol. 45, no. 2, pp. 299-305, Feb. 2000.

[10] H. H. Kha, H. D. Tuan, and H. H. Nguyen, "Fast global optimal power allocation in wireless networks by local D.C. programming," IEEE Trans. Wireless Commun., vol. 11, no. 2, pp. 510-515, Feb. 2012.

[11] J. Papandriopoulos and J. S. Evans, "SCALE: A low-complexity distributed protocol for spectrum balancing in multiuser DSL networks," IEEE Trans. Inf. Theory, vol. 55, no. 8, pp. 3711-3724, Aug. 2009.

[12] D. T. Ngo, S. Khakurel, and T. Le-Ngoc, "Joint subchannel assignment and power allocation for OFDMA femtocell networks," IEEE Trans. Wireless Commun., vol. 13, no. 1, pp. 342-355, Jan. 2014.

[13] L. Liu, R. Zhang, and K. C. Chua, "Wireless information and power transfer: a dynamic power splitting approach," IEEE Trans. Commun., vol. 61, no. 9, pp. 3990-4001, Sep. 2013.

[14] A. A. Nasir, D. T. Ngo, X. Zhou, R. A. Kennedy, and S. Durrani, "Joint resource optimization for multicell networks with wireless energy harvesting relays," ArXiv Technical Report, 2014. [Online]. Available: http://arxiv.org/abs/1408.4215

[15] S. Boyd and L. Vandenberghe, Convex Optimization. Cambridge University Press, 2004.

[16] X. Lu, P. Wang, D. Niyato, D. I. Kim, and Z. Han, "Wireless networks with RF energy harvesting: A contemporary survey," ArXiv Technical Report, 2014. [Online]. Available: http://arxiv.org/abs/1406.6470

[17] M. Grant and S. Boyd, "CVX: Matlab software for disciplined convex programming, version 2.1," http://cvxr.com/cvx, Mar. 2014. 